\title{
PRIMARY REPAIR OF CLEFT LIP AND NOSE IN THE NEONATAL PERIOD
}

\author{
Valentova Strenacikova $\mathbf{S}^{1}$, Malina R. ${ }^{2}$ \\ ${ }^{1}$ Clinic of Plastic, Aesthetic and Reconstructive Surgery, F. D. Roosevelt Hospital, \\ Banská Bystrica, Slovakia \\ ${ }^{2}$ Department of Biology and Ecology, Faculty of Natural Sciences, Matej Bel University, \\ Banská Bystrica, Slovakia
}

\begin{abstract}
Facial cleft malformations belong to the most common problems of the congenital facial anomalies. At the Clinic of Plastic, Aestetic and Reconstructive Surgery at the Faculty Hospital in Banská Bystrica we have been performing primary cleft lip repairs during the first week of life of a newborn since 1995. During this life period the fetal healing still persists. Modern anesthetic techniques have considerably reduced respiratory complications after the lip closure. The adequate postoperative sedation together with monitoring of the pacient remarkably reduces the occurrence of wound dehiscence. The advantages of the neonatal cleft lip repair are the scarless healing, no negative effect on the growth of the middle thirt of the face, as well as the pschychosocial effect on the family and the fact that the ortodontic aparat has not been needed.
\end{abstract}

Key words: cleft lip, cleft palate, craniofacial malformation, scarless healing, newborn

\section{INTRODUCTION}

The corrections of the lip in the neonatal period started in a close cooperation of the Clinic of Plastic, Aesthetic and Reconstructive Surgery (CPARS) and the Children's Faculty Hospital in Banska Bystrica in 1995. Globally, the cleft centers are operating on approaches to solve cleft lip from the first hour after the birth to the adulthood (1). Nowadays two concepts (early and late operational solution) are promoted.

In 1966 Musgrave and Wilhemsen (2) published a study in which they suggested an operative solution in age of 3 months (and so 10 pounds in weight, 10 grams for $\mathrm{Hb} \%, 10$ week for age). Most children, however, do not reach the required amount of $\mathrm{Hb}$ or weight even in the age of three months; in such case the surgical reconstruction is postponed.

On the other hand, the other group advocates a trend of early surgical techniques. Surgical reconstruction of the primary palate is possible already during the first week after the birth (3).

One of the central counter-arguments is the risk of anesthesia in the newborn. Establishment of pediatric anaesthesiology as a new subspecialty followed by acceleration of development in this field of medicine considerably minimizes that risk. There has been noticeable decrease in the occurrence of perioperative complications since the mid $20^{\text {th }}$ century, mainly due to the pharmacological and technological development. It is associated with the evolution of anesthetic equipment allowing controlled ventilation of all weight categories. At the same time a pulse oximeter and a kapnometer were introduced to standard perioperative monitoring. Nowadays, advances in neonatology and pediatric anestesia allow to perform the cleft surgery during the neonatal period (4).

Address for correspondence:

Silvia Valentova, MD, Clinic of Plastic, Aesthetic and Reconstructive Surgery; Hospital F. D. Roosevelt, Nám. L. Svobodu 1, 97517 Banská Bystrica, Slovakia

E - mail: silviavs@atlas.sk 
Cleft lip and/or palate (CL/P) is the most common craniofacial malformation in Slovakia (1.5-1.8 per 1,000 live births). Statistically it is the $9^{\text {th }}$ most common of all congenital malformations in Slovakia (5). There is not only the case of simple unrelated tissues but also hypoplasia or insufficiency of individual anatomical structures that can occur with varying severity. Cleft lip and palate can occur as an isolated form but also in association with other congenital malformations. The complete repair includes the creation of an intact and appropriately sized upper lip to compensate loss of philtrum height on the cleft side, repair of muscular structure, and primary repair of nasal deformity in order to achieve the required goal (6). The medical and psychological problems of diagnosis affect both the child and the whole the family.

Prenatal diagnosis is focused on the sonographic examination. The structures of the central part of the fetal face should completely within the $7^{\text {th }}$ week of gestation; mandible and maxilla are not clearly visualized up to 10 weeks. CL/P is reliably diagnosed by transabdominal sonography and transvaginal sonography in the $13-14^{\text {th }}$ week. Fetal palate is best seen in the axial direction; fetal lip is best visualized in the coronal view. The opening in the maxilla and the palate is visible in the transverse direction. In the second trimester the transabdominal sonography is suitable for prenatal diagnosis (7).

At the CPARS we approach operational solving of lips in neonatal period during the first 3 weeks of life. Surgical intervention is carried out before discharging the newborn from the hospital. We have had no increase in mortality and morbidity in comparison with the solution performed after the age of 3 months. The scars are very slight, especially in children with surgical correction of the lips and nose wings in the period from 48 hours to 7 days after birth $(8,9)$.

\section{THE CLINICAL MATERIAL}

It consists of our young patients with surgical correction of lip performed from 1995 to 2016, all with diagnosis of cleft malformations, either as the primary palate or as the primary and secondary palate unilaterally/bilaterally. Included are not only the early operating solutions but also the later (due to either associated congenital disease, ignorance of parents or they will). The children were of various types of clefts (unilateral, complete, incomplete, severe bilateral cleft). For surgical correction we followed our surgical protocol.

The exclusion criteria for patient selection were: infant with other congenital anomalies that affect life or interfere with anesthesia (e.g. cardiac, renal, hydrocephalus, etc.), infants with body weight less than two kilograms.

We evaluated the scar but we focused on the associated complications in terms of surgical and anesthesiological also. The child was investigated by a pediatrician, an anesthetist, followed by cardiac examination, genetic testing, together with a detailed history of the parents, psychological consultation, and examination of jaw orthodontics together with the making of the maxillary dental casts before surgical lips correction $\left(1^{\text {st }}\right.$ operational phase of the solution). This was realized before the palate correction at the age of 6-8 months. On this basis, we can clearly see the effect of early correction of dentoalveolar arch, the adequate formation of the orbicularis oris deductibles. The surgical intervention was always performed under a microscope.

\section{RESULTS}

At the CPARS there were 571 operated patients, the effect of sample operation is shown in figure (Fig. 1a-b). Types of cleft malformations are shown in the Table 1 . The most common was occurrence of left-sided unilateral cleft lip associated with cleft palate (45.8\%). There was also severe bilateral cleft. Early operational solution of cleft lip one week after the birth involves a whole set of 269 children (56.5\%), later 97 children (17\%); see the Table 2. Surgical technique and the procedure is shown in Fig. 2 a,b,c. 


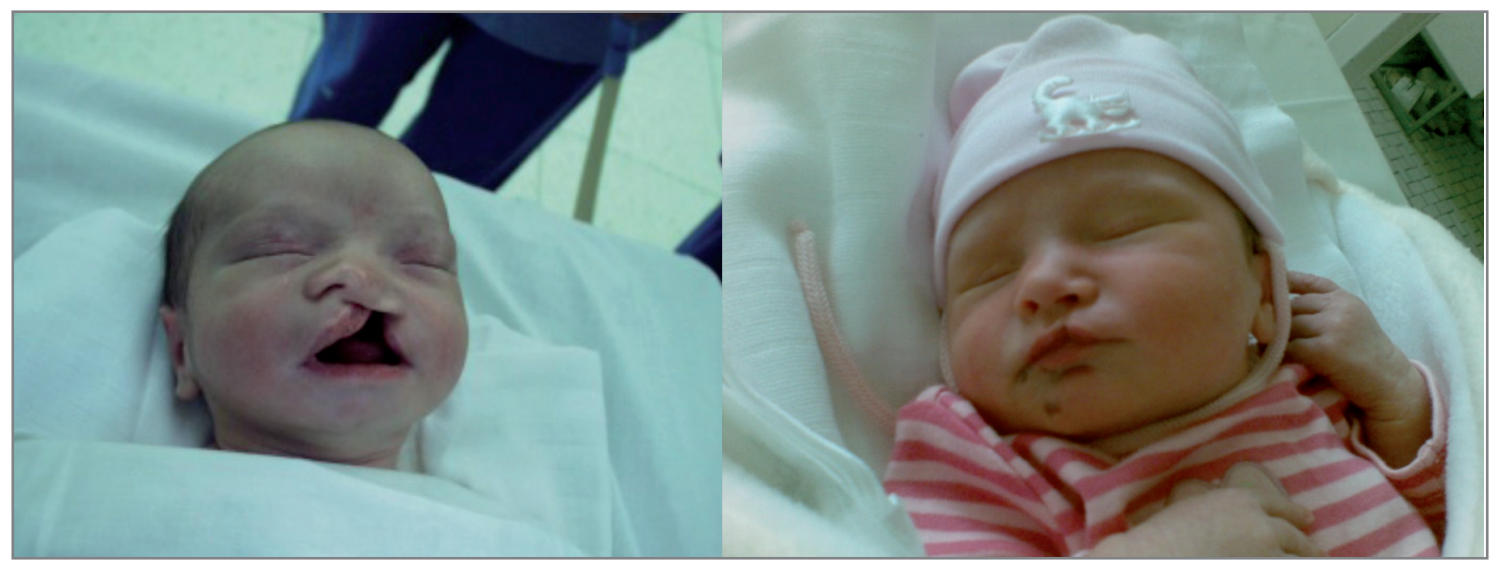

Fig. 3 a - Child with a severe bilateral cleft lip and palate; b - Child with orthodontic appliances; $\mathbf{c}$ - Total endotracheal intubation with infraorbital block

Tab. 1 Types of cleft malformations

\begin{tabular}{|c|c|c|c|}
\hline \multicolumn{2}{|c|}{ Unilateral clefts } & Bilateral clefts & Middline clefts \\
\hline Only primary palate & Primary + secondary palate & & \\
\hline 153 & 194 & 106 & 118 \\
\hline
\end{tabular}

Tab. 2 Early versus later operational solution of the cleft lip

\begin{tabular}{|l|c|c|}
\hline Early & 474 & $\mathbf{8 3} \%$ \\
\hline Perinatal $\left(1^{\text {st }}\right.$ week $)$ & 269 & $56.5 \%$ \\
\hline Early $\left(8^{\text {th }}\right.$ day $-3^{\text {rd }}$ week $)$ & 205 & $26.5 \%$ \\
\hline Later & 97 & $17 \%$ \\
\hline Later (3 ${ }^{\text {rd }}$ week $\left.-3^{\text {rd }}\right)$ & 83 & $2.5 \%$ \\
\hline Late (more than 3 months $)$ & 14 & 100 \\
\hline Total & 571 & $2.5 \%$ \\
\hline
\end{tabular}

In unilateral cleft the Millard operating technique is preferred. Its aim is the functional and anatomical reconstruction of a lip, the reconstruction of the nasal suture entrance with nasal septal mucoperiosteal flap, correction of nasal deformity, nasal base media approach, and straightening columella.

In bilateral cleft lip we preferred operational methodology according to Black. In case of a very severe cleft it is important to consult an orthodontist about using of the orthodontic appliances. The primary goal of presurgical orthodontic treatment is to realign the maxillary segments to achieve a more normal position of the segment and the alveolar arch, as well as a more symmetric skeletal base. 

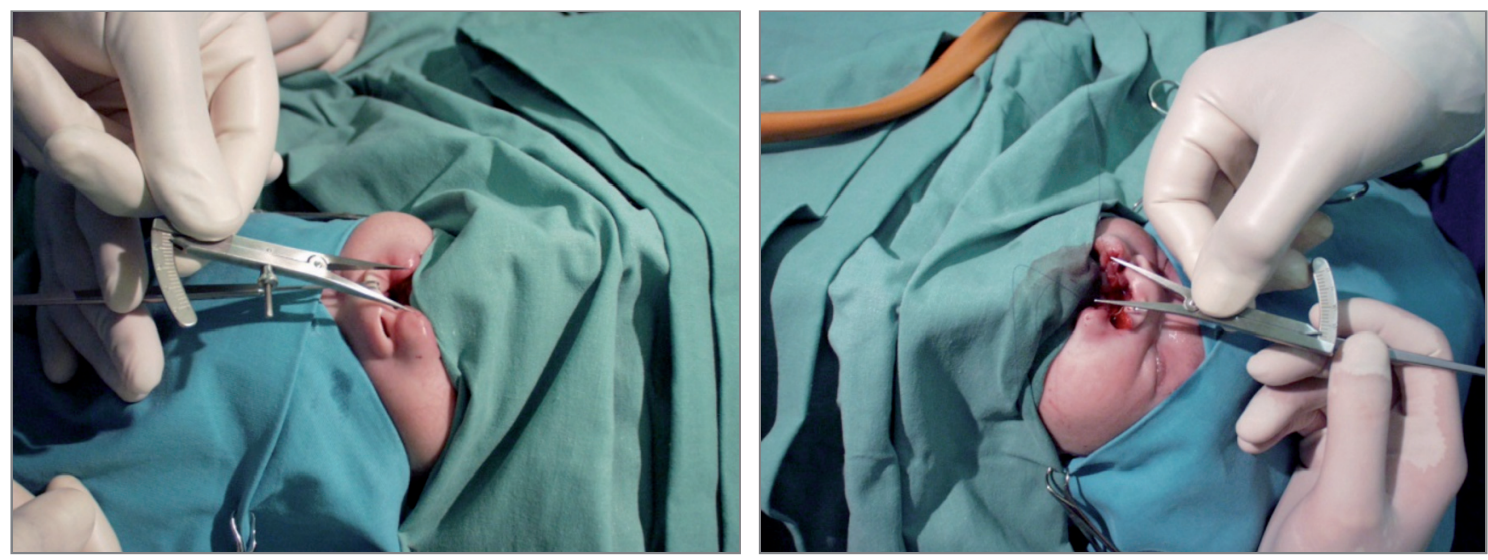

Fig. 2 a, b - Preoperative measurement and incision; c - Child after the surgery

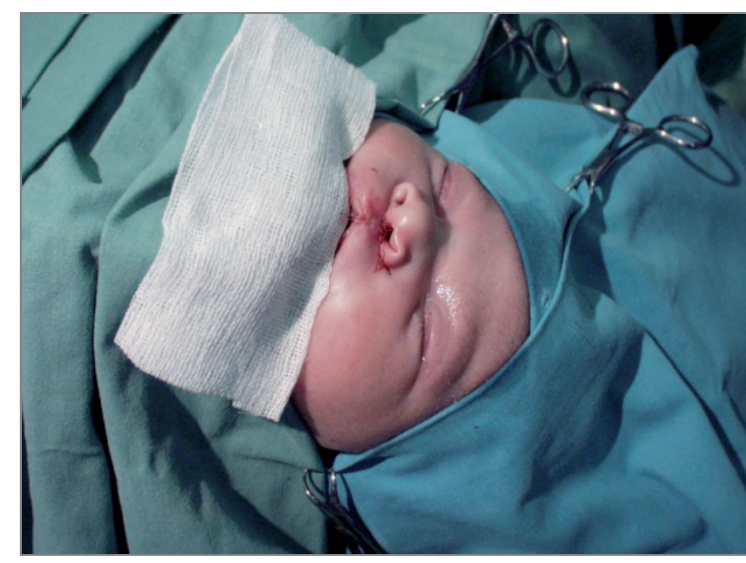

In case of a jaw-orthodontic apparatus the primary goal is reorganization of dentoalveolar segments to achieve their optimal position. In terms of our anesthetic workplace endotracheal intubation with controlled ventilation is preferred, which is also preferably combined with infraorbital block (Fig. 3a, b, c).

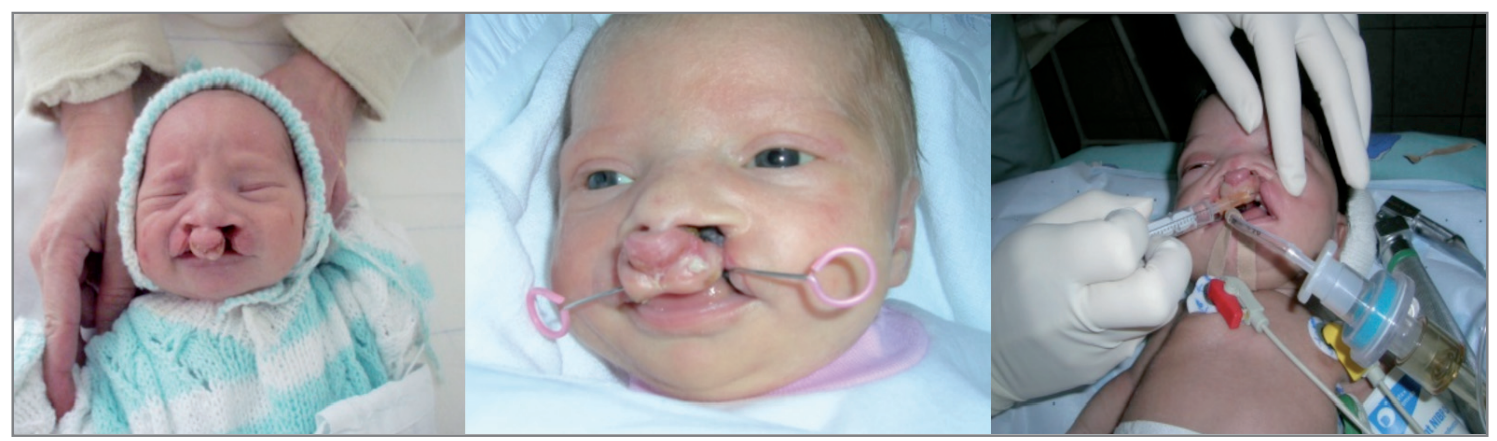

Fig. 3 a - Child with a severe bilateral cleft lip and palate; b - Child with orthodontic appliances; $\mathbf{c}$ - Total endotracheal intubation with infraorbital block 
Successful implementation of infraorbital block eliminates the administration of opioids during the surgical procedure and also substantially reduces the risk of postoperative apnea, respectively hypoventilation. At the end of surgery, with spontaneous respiratory activity, the child can be extubated in the operating room, followed by $1-2$ days of the sedation (combined Tramal - Dormicum) which is individual (Fig. 4). The day after the surgery begins with oral nutrition.

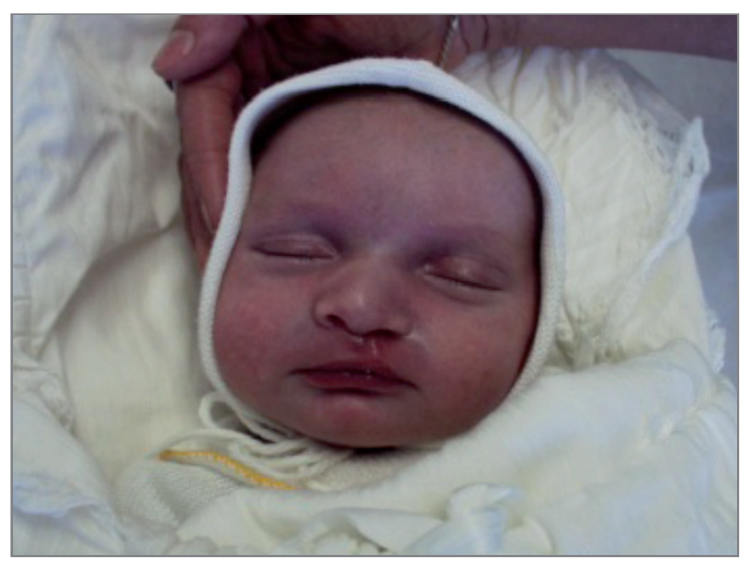

Fig. 4 Child after the surgery in analgosedation

The most common surgical complications include a small wound dehiscence ( 4 cases) and complete wound dehiscence ( 2 cases). Anesthetic complications are aspiration pneumonia (4 cases) and severe respiratory depression (1 case). The total duration of hospitalization requires 4 to 5 days.

\section{DISCUSSION}

Based on a comparison of results of children with surgical intervention in the neonatal period and after 3 months, we concluded that the early surgical intervention has several advantages. The scar is almost negligible, individual tissues are more formable, and there is also a huge family psychosocial benefit. The correction of lips usually adjusted better feed intake, weight gain was identical to noncleft children, and also middle ear infections were significantly reduced. Early correction reduces the use of ortodontic appliance, which is important for optimal molding of alveolar segments. Ortodontic appliance was used only in cases of severe bilateral clefts.

There are many opinions on many scientific conferences and clinical trials of presurgical orthodontic treatment in the neonatal period, or up to the period before the surgical intervention lip reconstruction. Based on the results of many studies the scientific evidence concludes that POT has a lasting positive impact on the growth of the dentoalveolar arch (10) The issue of using POT with cleft malformations remains largely unresolved problem. In our group of patients presurgical ortopedic therapy was not used in either case. The function took upgraded orbicularis oris that ensured the proper formation of dentoalveolar arch and cleft closure. Based on the results we can say that the reduction of alveolar cleft width (GL) comes just after the lip reconstruction. After the reconstruction of muscular system the orbicularis oris muscle operates the molding effect on noncleft segment and enhances the mutual position of the alveolar segments. The same effect was also recorded in Thierens et al. (2017), Eichhorn et al. (2011) and Christie et al. (1991) studies (15, 11, 12).

The results of our study confirm that early surgical reconstruction of lips up to 7 days after the birth has a positive effect on the growth and development of the anterior segment of the dentoalveolar arch. Early reconstruction of lip forms a continuous pressure on the 
front segment, which leads to a faster adjustment of anatomical properties and creates favorable conditions for the development of the best functional and aesthetically very important area (13). In the period until the first year of the life we did not notice any negative effect on the growth and development of anterior alveolar segment. Early surgical reconstruction significantly improves the aesthetics of the middle third of the face, eating, swallowing and, of course, correct language development. Evaluation of the effectiveness of the treatment process is one of the goals of our long-term monitoring program of growth and development of the front, rear, and alveolar segment of the middle third of the face in patients with complete unilateral cleft malformations. In patients with early surgical management the monitoring and evaluation of the growth and development of front, rear, and alveolar segment of the middle third of the face from birth to adulthood will be set.

In the past decade the lip repair in the first neonatal days arose as a possible trend to achieve a better infant-mother relationship with positive psycho-social effect (14).

\section{CONCLUSION}

In the last two decades there has been a substantial progress in the management of cleft patients. A comprehensive multidisciplinary care for children with cleft includes reintroduction into normal life, achievement of mental stability and high self-esteem. Early surgical intervention significantly contributes to improving the appearance of young patients and greatly improves quality of life. At the same time we also concluded that early surgery reduces the number of secondary corrections. The result of this greater effectiveness for the patient and economically more advantageous treatment gives priority to picking multidisciplinary cooperation in cleft patients treatment which lasts from the birth until the adulthood.

\section{REFERENCES}

1) Millard DR. Cleft Craft: The evalution of Its Surgery, vol. 1. Boston, Little, Brown, 1976.

2) Wilhemsen HR, Musgrave RH. Complications of cleft lip surgery. Cleft palate 3, 1996, p. 223.

3) Gary S, Bromley M, Kenneth O, Rothaus M, Dicrano G. Cleft Lip, Mortality and Morbidity in Early Repair. Ann Chir Plast 1983 1:214-17.

4) Sandberg DJ, Magee WP, Denk MJ. Neonatal cleft lip and cleft palate repair. AORN J 2002 75(3): 490-8; quiz 501-4, 506-8.

5) Kokavec R, Hedera J, Fedeles J, Janovic J, Short E, Klimová I. New Trends in the complex treatment in the Cleft Centre in Bratislava. Bratisl Lek Listy 2001 102(6):290-3.

6) Mahdi A., Shkoukani MC, Vong A. Cleft Lip - A Comprehensive Review. Front Pediatr. 2013; 1(1):53.

7) Cockell A, Lees M. Prenatal diagnosis and management of orofacial cleft. Prenatal Diagnosis 2000, 20:149-51.

8) Weinfeld AB, Hollier LH, Spira M, Stal S. International trends in treatment of cleft lip and palate. Clin Plast Surg 2005 32:19-23.

9) Borsky J, Kozak J, Tvrdek M, Hudáček M, Dostalova T, Černý M. Cleft defect in the upper lip. Czech journal of dental chambers 2007, 11:18-21.

10) Sunjay S, Tompson, BD. A Modified Muscle-Activated Maxillary Orthopedic Appliance for Presurgical Nasoalveolar Molding in Infants With Unilateral Cleft Lip and Palate. The Cleft PalateCraniofacial Journal 2004, 41(3):225-27.

11) Christie FB, Stirrups DR, Mackenzie JS, Lawrence GM. An orthodontic evaluation of 16-year-old males with an original complete unilateral cleft lip and palate problem repaired during the neonatal period. British Journal of Plastic Surgery 1991, 44:557-61.

12) Eichhorn W, Blessmann M, Vorwig O, Gehrke G, Schmelzle R, Heiland M. Influence of lip closure on alveolar cleft width in pacient with cleft lip and palate. Head \& Face Medicine 2011, 7(3).

13) Hammoudeh, JA, Imahiyerobo TA, Liang F, Fahradyan, A, Urbinelli, L, Lau J, Matar M, Magee W, Urata M. Early Cleft Lip Repair Revisited: A Safe and Effective Approach Utilizing a Multidisciplinary Protocol. Plastic and Reconstructive Surgery 2017, 5(6):1340. 
14) Eltramsy EHEA, Barakat A. Early Cleft Lip Repair. AAMJ 2012, 10(1):317-22.

15) Thierens LAM, Brusselaers N, De Roo NMC, De Pauw GAM. Effects of labial adhesion on maxillary arch dimensions and nasolabial esthetics in cleft lip and palate: a systematic review. Oral Diseases 2017, 23(7):889-896.

Received: May, 23, 2018

Accepted: June, 26, 2018 